\title{
Two-Stage Autocorrelation Approach for Accurate Single Sinusoidal Frequency Estimation
}

\author{
Kenneth W. K. Lui and H. C. So \\ Department of Electronic Engineering, City University of Hong Kong, Kowloon, Hong Kong \\ email: 50469990@student.cityu.edu.hk, hcso@ee.cityu.edu.hk
}

\begin{abstract}
A two-stage autocorrelation approach is proposed for single-tone frequency estimation of a real sinusoid in white noise. In the first stage, we transform the received data to another noisy sinusoidal sequence of same frequency via an autocorrelation procedure. Autocorrelation functions of the converted sequence are then employed for frequency estimation in the second stage. A simple frequency estimator is first derived, followed by a generalized algorithm. Computer simulations are included to illustrate the accuracy of our proposed approach via comparison with Cramér-Rao lower bound and several conventional frequency estimators.
\end{abstract}

\section{Index Terms}

Frequency estimation, single sinusoid, autocorrelation

\section{INTRODUCTION}

The problem of frequency estimation of a single real sinusoid in white noise has received much attention in the literature [1]. The discrete-time signal model for single-tone frequency estimation is

$$
x(n)=s(n)+q(n), \quad n=1,2, \cdots, N
$$

where

$$
s(n)=\alpha \cos (\omega n+\phi)
$$

The $\alpha, \omega \in(0, \pi)$ and $\phi \in[0,2 \pi)$ are unknown but deterministic constants which represent the tone amplitude, frequency and phase, respectively, while the noise $q(n)$ is assumed to be a zero-mean white process with unknown variance $\sigma^{2}$. It is assumed that the number of samples of $\{x(n)\}$, namely, $N$, is at least 11 .

When $q(n)$ is Gaussian distributed, the maximum likelihood (ML) estimate of frequency [2] is obtained by maximizing a highly nonlinear and multi-modal cost function but extensive computations are involved. Apart from the ML estimator, other conventional frequency estimation techniques include iterative filtering algorithm (IFA) [3], 
Capon methods, autoregressive (AR) modeling [4], discrete Fourier coefficient (DFT) interpolation [5], Yule-Walker methods and subspace based approaches [6]- [9], and they are more computationally efficient but generally provide suboptimal estimation accuracy. For example, the Pisarenko harmonic decomposer (PHD) [6] exploits the eigenstructure of the covariance matrix for $x(n)$, and the reformed Pisarenko harmonic decomposer (RPHD) [7] utilizes the data matrix and is shown to be an improvement over the classical counterpart. While the MUSIC [8] and principal component (PC) [9] methods can give better estimation performance than [6]- [7] but in the expense of higher computational requirement. In this paper, we contribute to the development of an accurate single-tone frequency estimation approach based on linear prediction (LP) and autocorrelation.

The rest of the paper is organized as follows. In Section II, the key idea of our two-stage autocorrelation approach is proposed and a simple frequency estimator is devised. In the first stage, we convert $\{x(n)\}$ to another noisy sinusoidal sequence of same frequency via an autocorrelation procedure. Autocorrelation functions of the converted sequence with lags 1 and 2 are then utilize to estimate $\omega$ in the second stage. By examining the tradeoff between the maximum allowable numbers of data samples in the two stages of autocorrelation computation, a generalized and improved realization of the two-stage autocorrelation approach is derived in Section III. Numerical examples are included in Section IV to evaluate the performance of the proposed algorithms by comparing with that of the ML estimator, IFA, DFT interpolation, PHD, RPHD, MUSIC and PC methods, as well as Cramér-Rao lower bound (CRLB) [10]. Finally, conclusions are drawn in Section V.

\section{BASIC IDEA}

We start with the well known LP property in a single real tone:

$$
2 \cos (k \omega) s(n-k)=s(n)+s(n-2 k)
$$

Multiplying both sides of (3) by $s(n-k)$ yields

$$
2 \cos (k \omega) s^{2}(n-k)=s(n-k)[s(n)+s(n-2 k)]
$$

Based on (4), we define an autocorrelation-like function $\lambda_{k, M}$ where $k$ corresponds to the lag parameter:

$$
\lambda_{k, M}=\sum_{n=M+1+k}^{N-M+k} x(n-k)[x(n)+x(n-2 k)], \quad k=1,2, \cdots, M
$$

with $M=\left\lfloor\frac{N-1}{2}\right\rfloor \geq 5$ represents the largest integer contained in $\frac{N-1}{2}$. Unlike typical autocorrelation calculation, not all available samples are utilized in (5). More precisely, only three and four or six samples of $\{x(n)\}$ are involved in computing each $\lambda_{k, M}$ for odd and even $M$, respectively. With the use of (1) - (4), the expected value of (5) is easily shown to be

$$
E\left\{\lambda_{k, M}\right\}=2 \cos (k \omega) \sum_{n=M+1}^{N-M} s^{2}(n)
$$

where $E$ denotes the expectation operator. This means that $\left\{\lambda_{k, M}\right\}$ is also a noisy sinusoidal sequence with the same frequency of $\omega$ as in $\{x(n)\}$ but of different constant amplitude and phase, namely, $2 \sum_{n=M+1}^{N-M} s^{2}(n)$ and 0 , respectively. 
Due to the sinusoidal property of $\left\{\lambda_{k, M}\right\}$, we follow (5) to construct the second stage autocorrelation functions with lags 1 and 2 only, denoted by $\Lambda_{1, M}$ and $\Lambda_{2, M}$ :

$$
\Lambda_{1, M}=\sum_{k=4}^{M-1} \lambda_{k-1, M}\left(\lambda_{k, M}+\lambda_{k-2, M}\right)
$$

and

$$
\Lambda_{2, M}=\sum_{k=5}^{M} \lambda_{k-2, M}\left(\lambda_{k, M}+\lambda_{k-4, M}\right)
$$

Note that from (8), the minimum value of $M$ should be 5 which implies $N \geq 11$ is required. We have shown in the Appendix that for sufficiently large $N$ and when $\omega$ is neither close to 0 nor $\pi$, their expected values are

$$
E\left\{\Lambda_{1, M}\right\} \approx C_{M} \cos (\omega)
$$

and

$$
E\left\{\Lambda_{2, M}\right\} \approx C_{M} \cos (2 \omega)
$$

where $C_{M}=8 \alpha^{2}(M-4)\left[\alpha^{2}\left(N-2 M^{2}\right)+\sigma^{2}(N-8 M)\right]$. Cross-multiplying (9) and (10) with ignoring the expectation operator and employing $\cos (2 \omega)=\cos ^{2}(\omega)+1 / 2$, we obtain a quadratic equation for frequency which relates $\Lambda_{1, M}$ and $\Lambda_{2, M}$ :

$$
2 \Lambda_{1, M} \cos ^{2}\left(\hat{\omega}_{1}\right)-\Lambda_{2, M} \cos \left(\hat{\omega}_{1}\right)-\Lambda_{1, M}=0
$$

where $\hat{\omega}_{1}$ denotes the estimate of $\omega$ utilizing our basic idea of two-stage autocorrelation. Only one root of (11) corresponds to the actual frequency and it can be verified that $\hat{\omega}_{1}$ has the form of:

$$
\hat{\omega}_{1}=\cos ^{-1}\left(\frac{\Lambda_{2, M}+\sqrt{\Lambda_{2, M}^{2}+8 \Lambda_{1, M}^{2}}}{4 \Lambda_{1, M}}\right)
$$

From a theoretical aspect, the two-step approach is superior to the existing autocorrelation based methods because (i) The mean value of $\lambda_{k, M}$ is exactly equal to $2 \cos (k \omega) \sum_{n=M+1}^{N-M} s^{2}(n)$ while that of the standard autocorrelation is asymptotically equal to $2 \cos (k \omega)$. This means that the former will give a more accurate estimate of $\omega$ than the latter and (ii) Although not all available samples are used for computing a particular $\lambda_{k, M}$, all of them have been exploited in the whole set of $\left\{\lambda_{k, M}\right\}$. While typical autocorrelation-based methods employ all available samples for autocorrelation computation but only a few of them are utilized. For example, the PHD, which is analogous to (12), uses only the standard autocorrelations of $x(n)$ with lags 1 and 2 for frequency estimation. It is worthy to note that although both the proposed algorithm and [4] use sample covariances, the former considers the covariance sequence as another sinusoidal signal and utilizes (7)-(12) for frequency estimation. On the other hand, the covariances are employed in fitting an AR model and the frequencies are then determined using the roots of a polynomial constructed from the AR coefficients in the latter. Moreover, we apply a new sample covariance function proposed in (5) but [3] uses the standard one. 


\section{IMPROVED ESTIMATOR}

An obvious drawback in the above two-stage autocorrelation approach is that too few samples of $\{x(n)\}$ are involved in computing each of the $\left\{\lambda_{k, M}\right\}$. In fact, more samples can be utilized if the length of the first-stage autocorrelation function is reduced to $m<M$. That is, we generalize (5):

$$
\lambda_{k, m}=\sum_{n=m+1+k}^{N-m+k} x(n-k)[x(n)+x(n-2 k)], \quad k=1,2, \cdots, m, \quad m=5,6, \cdots, M
$$

The expected value of $\lambda_{k, m}$ is easily obtained from (6) with the substitution of $M=m$. In a similar manner, the second stage autocorrelation functions with lags 1 and 2 are:

$$
\Lambda_{1, m}=\sum_{k=4}^{m-1} \lambda_{k-1, m}\left(\lambda_{k, m}+\lambda_{k-2, m}\right)
$$

and

$$
\Lambda_{2, m}=\sum_{k=5}^{m} \lambda_{k-2, m}\left(\lambda_{k, m}+\lambda_{k-4, m}\right)
$$

It is proved in the Appendix that $E\left\{\Lambda_{1, m}\right\} \approx C_{m} \cos (\omega)$ and $E\left\{\Lambda_{2, m}\right\} \approx C_{m} \cos (2 \omega)$ where $C_{m}=8 \alpha^{2}(m-$ 4) $\left[\alpha^{2}\left(N-2 m^{2}\right)+\sigma^{2}(N-8 m)\right]$. That is, a general form of the proposed estimator is to replace $M$ by $m$ in (12). Nevertheless, we observe from $(13)$ - (15) that there is indeed a tradeoff between the maximum allowable numbers of samples in the two stages of autocorrelation computation. If we use a smaller value of $m$, more samples of $\{x(n)\}$ are involved in computing $\left\{\lambda_{k, m}\right\}$ but fewer samples of $\left\{\lambda_{k, m}\right\}$ can be used to evaluate $\Lambda_{1, m}$ and $\Lambda_{2, m}$, and vice versa. Although it is not trivial to determine the optimal $m$ in a theoretical way, we have experimentally determined that its optimal value with least computational requirement is approximately at $m=\lfloor M / 2\rfloor+5$.

As all $E\left\{\Lambda_{1, m}\right\}$ and $E\left\{\Lambda_{2, m}\right\}$ are proportional to $\cos (\omega)$ and $\cos (2 \omega)$, respectively, a simple improvement to the proposed approach is to utilize all $\left\{\Lambda_{1, m}\right\}$ and $\left\{\Lambda_{2, m}\right\}$ by summing them up to form:

$$
\Lambda_{1}=\sum_{m=5}^{M} \Lambda_{1, m}
$$

and

$$
\Lambda_{2}=\sum_{m=5}^{M} \Lambda_{2, m}
$$

where $E\left\{\Lambda_{1}\right\} \approx 2 \cos (\omega) \sum_{m=5}^{M} C_{m}$ and $E\left\{\Lambda_{2}\right\} \approx 2 \cos (2 \omega) \sum_{m=5}^{M} C_{m}$. With the use of (11) and (12), the improved estimate of $\omega$, denoted by $\hat{\omega}_{2}$, is then:

$$
\hat{\omega}_{2}=\cos ^{-1}\left(\frac{\Lambda_{2}+\sqrt{\Lambda_{2}^{2}+8 \Lambda_{1}^{2}}}{4 \Lambda_{1}}\right)
$$

Finally, we examine the computational complexity of the proposed approach and the results are tabulated in Table 1. Here $\hat{\omega}_{1}$ is generalized for $m=5,6, \cdots, M$, where its complexity is on the order of $m N$ for sufficiently large $N$. In particular, it can be deduced that calculating $\hat{\omega}_{1}$ with $m=M$ requires $(5 N-26)$ additions/substractions and $(3 N-10)$ multiplications when $N$ is even while $(3 N-19)$ additions/substractions and $(2 N-6)$ multiplications are needed if $N$ is odd. On the other hand, the complexity of $\hat{\omega}_{2}$ is on the order of $N M^{2}$. Roughly speaking, the computational requirements of $\hat{\omega}_{1}$ are comparable to those of the PHD [6] and RPHD [7] while $\hat{\omega}_{2}$, IFA [3], DFT interpolation [5], MUSIC [8] and PC [9] methods have similar complexity. 


\begin{tabular}{|c|c|c|c|c|c|}
\hline Method & Addition / Substraction & Multiplication & Division & Square Root & $\cos ^{-1}()$ \\
\hline \hline$\hat{\omega}_{1}$ & $4 m N-8 m^{2}+2 m-16$ & $2 m N-4 m^{2}+2 m-3$ & 1 & 1 & 1 \\
\hline$\hat{\omega}_{2}$ & $2 N M^{2}+M^{2}-\frac{8 M^{3}}{3}-32 N+224$ & $N M^{2}+M^{2}-\frac{4 M^{3}}{3}-\frac{26 M}{3}-16 N+104$ & 1 & 1 & 1 \\
\hline
\end{tabular}

Table 1: Computational complexity of proposed approach

\section{Simulation Results}

Computer simulations have been conducted to evaluate the sinusoidal frequency estimation performance of the proposed two-stage autocorrelation approach in the presence of white Gaussian noise by comparing with the ML estimator [2], IFA [3], DFT interpolation [5], PHD [6], RPHD [7] MUSIC [8] and PC [9] methods, as well as CramérRao lower bound (CRLB) [10]. The tone amplitude is fixed to $\sqrt{2}$ and we properly scale $q(n)$ to obtain different signal-to-noise ratios (SNRs) where $\mathrm{SNR}=\alpha^{2} /\left(2 \sigma^{2}\right)$. Unless stated otherwise, the number of samples is chosen as $N=100$, the sinusoidal phase is $\phi=0$ and $\mathrm{SNR}=20 \mathrm{~dB}$. All simulation results provided are averages of 1000 independent runs.

Figures 1 and 2 shows the mean square frequency errors (MSFEs) of different frequency estimators as well the CRLB versus $\omega$. The results of relatively low complexity algorithms, namely, $\hat{\omega}_{1}$, PHD and RPHD are provided in Figure 1 and those of the remaining methods are presented in Figure 2. As expected, the estimation accuracy of $\hat{\omega}_{2}$ of (18) is higher than that of $\hat{\omega}_{1}$ of (12). Moreover, it is observed that the former estimation performance is nearly uniform for $\omega \in[0.1 \pi, 0.9 \pi]$ and close to the CRLB in this range. On the other hand, (12) does not provide uniform MSFEs and its performance is degraded from the CRLB by around 11 to $20 \mathrm{~dB}$ when $\omega$ is neither near 0 nor $\pi$. We also see that the proposed approach of (18) is superior to all other frequency estimators except the ML method and IFA, while the estimator of (12) and RPHD have comparable performance and outperform the PHD. Furthermore, we have found that the two proposed estimators are nearly unbiased except for (12) at $\omega<0.06 \pi$ and $\omega>0.95 \pi$.

Figure 3 shows the MSFE performance of $\hat{\omega}_{2}$, ML, IFA, DFT interpolation, MUSIC and PC methods versus $\phi \in$ $[0,2 \pi)$ at $\omega=0.3 \pi$. It is observed that their estimation accuracy is nearly independent of $\phi$. We also see that the performance of (18) is comparable with the ML estimator and IFA while other observations are similar to those of Figure 2. Figures 4 and 5 show the MSFE performance versus SNR and $N$, respectively, at $\omega=0.3 \pi$, and similar findings are obtained. Finally, the performance of the proposed estimator with our suggested optimum $m$ is shown in Figure 6 where the simulation settings are identical to those of Figures 1 and 2. It is seen that its estimation accuracy only deviates from that of $\hat{\omega}_{2}$ within $1 \mathrm{~dB}$ when $\omega$ is neither close to 0 nor $\pi$.

\section{CONCLUSION}

A two-step approach has been proposed for accurate frequency estimation of a single real sinusoid in additive white noise. In the first step, we convert the received data to another noisy sinusoidal sequence of same frequency via an autocorrelation process. The second step is to utilize the autocorrelation functions of the converted sequence with lags 1 and 2 to produce the frequency estimate. Two versions of the proposed approach, namely, a simple algorithm and then an improved algorithm, are devised. It is shown that the performance of the improved method can attain Cramér-Rao lower bound for different data lengths, signal-to-noise ratios, sinusoidal frequencies and phases. 


\section{APPENDIX}

The expected values of $\Lambda_{1, m}$ and $\Lambda_{2, m}, m=5,6, \cdots, M$, when $N$ is sufficiently large and $\omega$ is neither close to 0 nor $\pi$, are now derived as follows. Define $f(k, d)=E\left\{\lambda_{k, m} \lambda_{k-d, m}\right\}$, we have

$$
E\left\{\Lambda_{1, m}\right\}=\sum_{k=4}^{m-1}[f(k, 1)+f(k-1,1)]=\sum_{k=4}^{m-1} f(k, 1)+\sum_{k=3}^{m-2} f(k, 1)
$$

and

$$
E\left\{\Lambda_{2, m}\right\}=\sum_{k=5}^{m}[f(k, 2)+f(k-2,2)]=\sum_{k=5}^{m} f(k, 2)+\sum_{k=3}^{m-2} f(k, 2)
$$

The term $f(k, d)$ is evaluated as

$$
\begin{aligned}
f(k, d)= & 4 \cos (k \omega) \cos ((k-d) \omega)\left(\sum_{n=m+1}^{N-m} s^{2}(n)\right)^{2}+4 \sigma^{2} \cos (k \omega) \cos ((k-d) \omega)\left(\sum_{n=m+1}^{N-m} s^{2}(n)\right)+ \\
& \sigma^{2}\left(\sum_{n=m+1}^{N-m-d} s(n) s(n+d)+\sum_{n=m+1}^{N-m-2 k+d} s(n) s(n+2 k-d)+\right. \\
& \left.\sum_{n=m+1-d}^{N-m} s(n) s(n-2 k+d)+\sum_{n=m+1+2 k+d}^{N-m} s(n) s(n-d)\right)+ \\
& 2 \sigma^{2} \cos (k \omega)\left(\sum_{n=m+1+k-d}^{N-m} s(n) s(n-k+d)+\sum_{n=m+1}^{N-m-k+d} s(n) s(n+k-d)\right)+ \\
& 2 \sigma^{2} \cos ((k-d) \omega)\left(\sum_{n=m+1+k}^{N-m} s(n) s(n-k)+\sum_{n-m+1}^{N-m-k} s(n) s(n+k)\right)
\end{aligned}
$$

For sufficiently large $q$, we have:

$$
\sum_{n=p}^{q} s(n) s(n+l)=\frac{\alpha^{2}}{2}(q-p+1) \cos (l \omega)+\frac{\alpha^{2}}{2} \sum_{n=p}^{q} \cos ((2 n+l) \omega+2 \phi) \approx \frac{\alpha^{2}}{2}(q-p+1) \cos (l \omega)
$$

With the use of (A.4), (A.3) is approximated as

$$
f(k, d) \approx 4 \alpha^{2} \cos (k \omega) \cos ((k-d) \omega)\left(\alpha^{2}(N-2 m)^{2}+\sigma^{2}(N-8 m-3 k)\right)+\alpha^{2} \sigma^{2}(-2 k+4 d) \cos ((2 k-d) \omega)
$$

Summing $f(k, d)$ from $k=p$ to $k=q$ yields

$$
\begin{aligned}
\sum_{k=p}^{q} f(k, d) & \approx 4 \alpha^{2} \sum_{k=p}^{q} \cos (k \omega) \cos ((k-d) \omega)\left(\alpha^{2}(N-2 m)^{2}+\sigma^{2}(N-8 m-3 k)\right) \\
& \approx 4 \alpha^{2} \cos (d \omega)(q-p+1)\left(\alpha^{2}(N-2 m)^{2}+\sigma^{2}(N-8 m)\right)
\end{aligned}
$$

Employing (A.6), (A.1) and (A.2) can be simplified as

$$
E\left\{\Lambda_{1, m}\right\} \approx \cos (\omega) \times 8 \alpha^{2}(m-4)\left[\alpha^{2}\left(N-2 m^{2}\right)+\sigma^{2}(N-8 m)\right]
$$

and

$$
E\left\{\Lambda_{2, m}\right\} \approx \cos (2 \omega) \times 8 \alpha^{2}(m-4)\left[\alpha^{2}\left(N-2 m^{2}\right)+\sigma^{2}(N-8 m)\right]
$$




\section{REFERENCES}

[1] P. Stoica and R. Moses, Spectral Analysis of Signals, Upper Saddle River, NJ : Prentice-Hall, 2005

[2] R.J. Kenefic and A.H. Nuttall, "Maximum likelihood estimation of the parameters of tone using real discrete data," IEEE Journal of Oceanic Engineering, vol.12, no.1, pp.279-280, 1987

[3] S.M. Kay, "Accurate frequency estimation at low signal-to-noise ratio," IEEE Trans. Acoust., Speech, Signal Processing, vol.32, no.3, pp.540547, June 1984

[4] J. Angeby, P. Stoica and T. Soderstrom, ”Asymptotic statistical analysis of autoregressive frequency estimates," Signal Processing, vol.39, pp.277-292, 1994

[5] B.G. Quinn, "Estimating frequency by interpolation using Fourier coefficients," IEEE Trans. Signal Processing, vol.42, no.5, pp.1264-1268, May 1994

[6] A. Eriksson and P. Stoica, "On statistical analysis of Pisarenko tone frequency estimator," Signal Processing, vol.31, no.3, pp.349-353, 1993

[7] H.C. So and K.W. Chan, "Reformulation of Pisarenko harmonic decomposition method for single-tone frequency estimation," IEEE Trans. on Signal Processing, vol.52, no.4, pp.1128-1135, April 2004

[8] P. Stoica and A. Eriksson, "MUSIC estimation of real-valued sine-wave frequencies," Signal Processing, vol.42, pp.139-146, 1995

[9] S.M. Kay and A.K. Shaw, "Frequency estimation by principal component AR spectral estimation method without eigendecomposition," IEEE Trans. Acoust., Speech, Signal Processing, vol.36. no.1, pp.95-101, Jan. 1988

[10] S.M. Kay, Fundamentals of Statistical Signal Processing: Estimation Theory, Englewood Cliffs, NJ: Prentice-Hall, pp.56-57, 1993

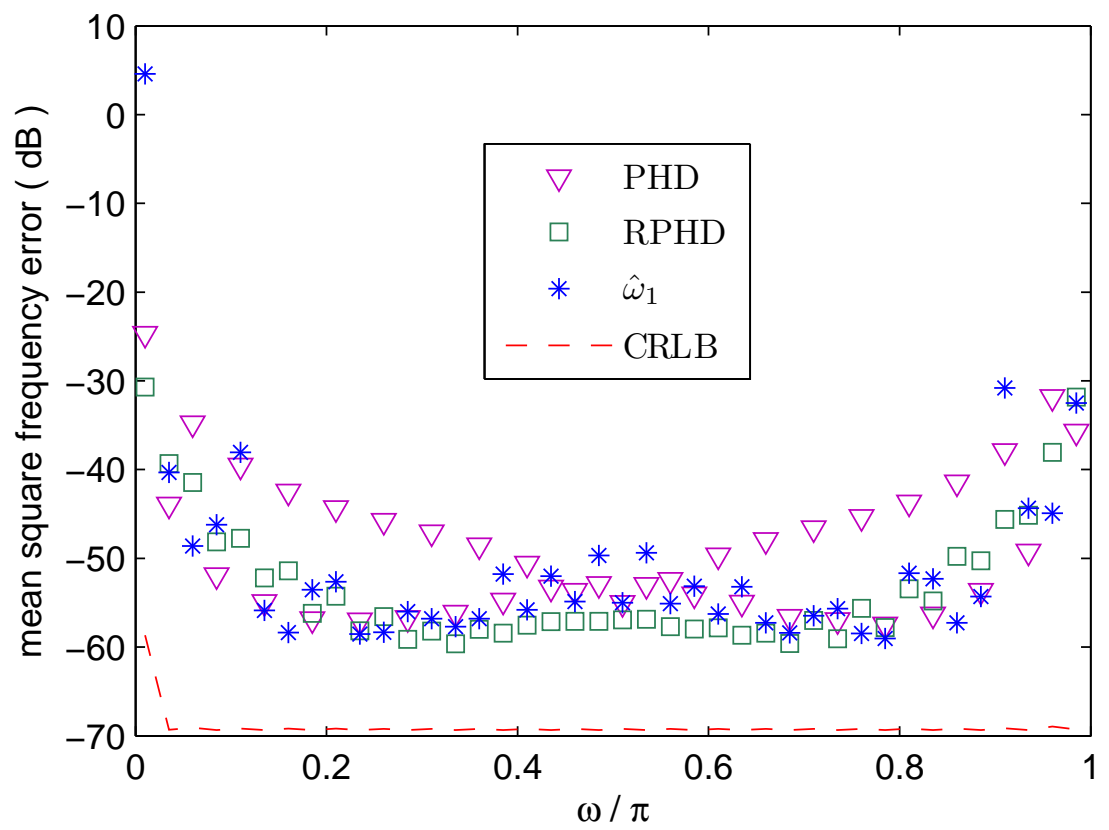

Fig. 1. Mean square frequency error versus $\omega$ for $\hat{\omega}_{1}$ 


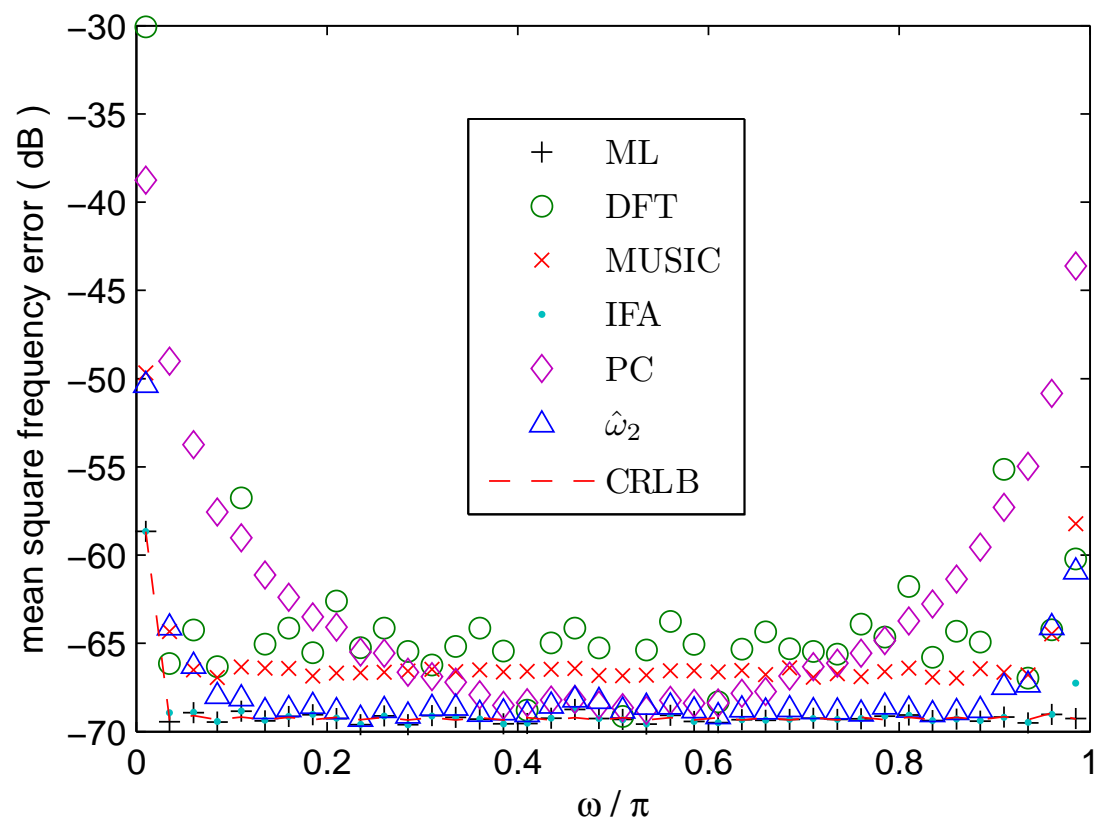

Fig. 2. Mean frequency error versus $\omega$ for $\hat{\omega}_{2}$

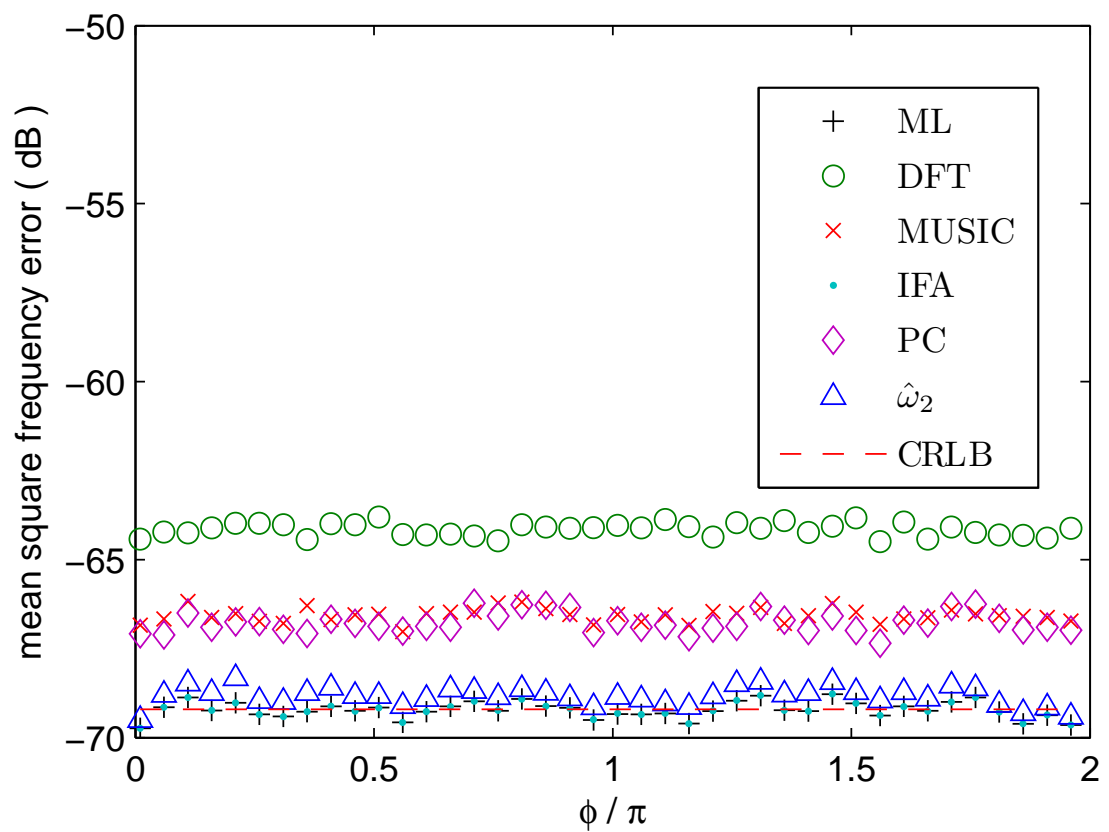

Fig. 3. Mean square frequency error versus $\phi$ at $\omega=0.3 \pi$ 


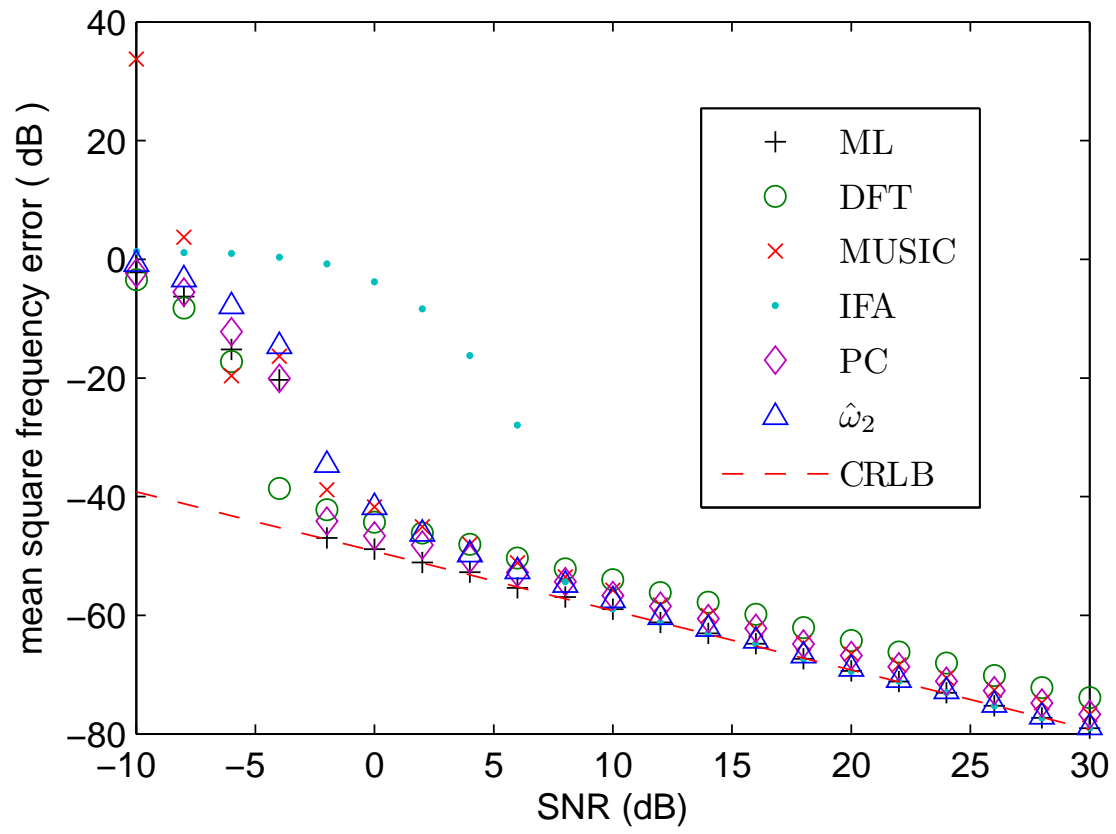

Fig. 4. Mean square frequency error versus SNR at $\omega=0.3 \pi$

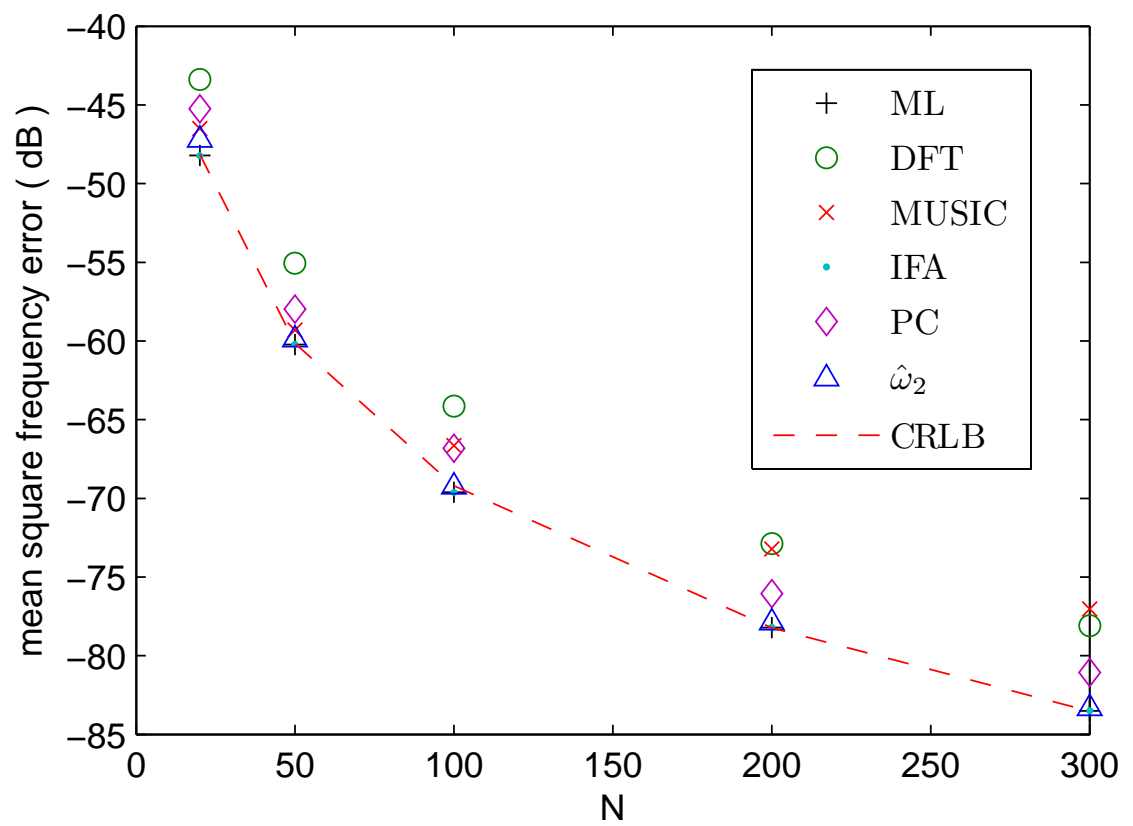

Fig. 5. Mean square frequency error versus $N$ at $\omega=0.3 \pi$ 


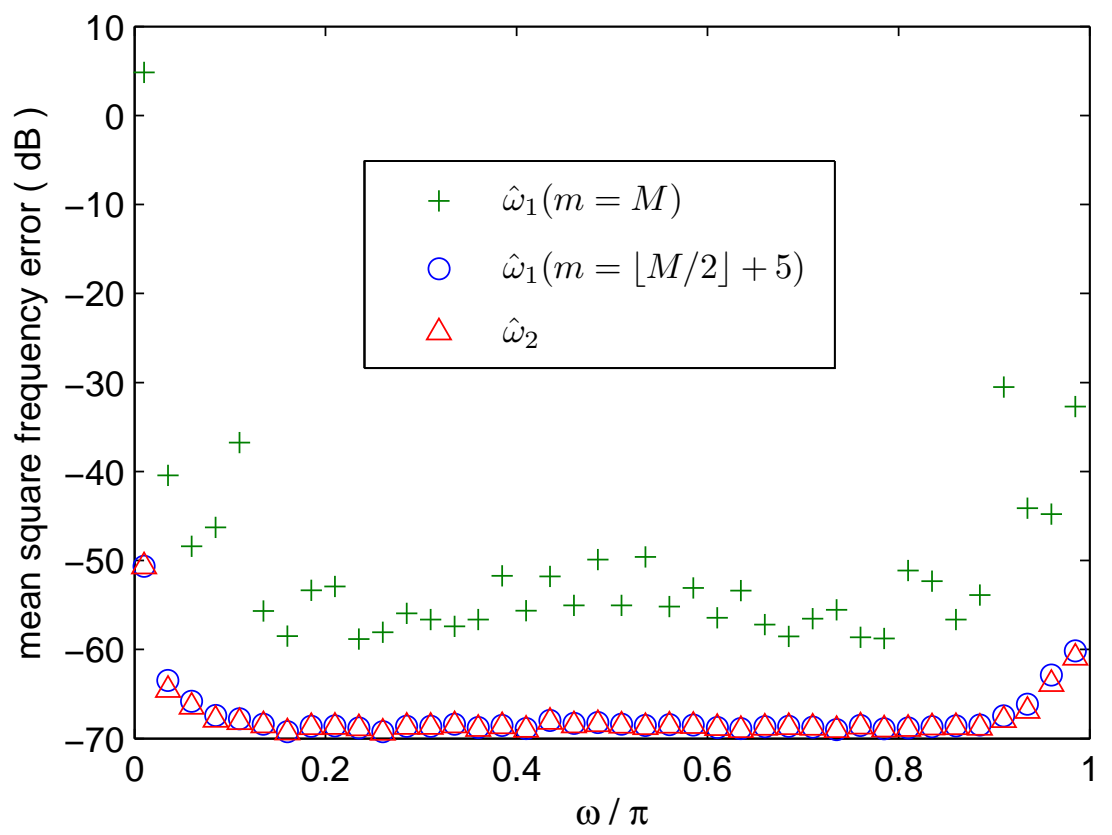

Fig. 6. Mean square frequency error versus $\omega$ with optimum $m$ 